\title{
Questes
}

vestes Revue pluridisciplinaire d'études médiévales

11 | 2007

La transmission

\section{Les épées du roi Arthur}

\section{Patrick Moran}

\section{(2) OpenEdition}

Journals

Édition électronique

URL : https://journals.openedition.org/questes/581

DOI : 10.4000/questes.581

ISSN : 2109-9472

\section{Éditeur}

Les Amis de Questes

\section{Édition imprimée}

Date de publication : 15 mars 2007

Pagination : 26-35

ISSN : 2102-7188

\section{Référence électronique}

Patrick Moran, «Les épées du roi Arthur », Questes [En ligne], 11 | 2007, mis en ligne le 01 janvier 2014 consulté le 21 septembre 2021. URL : http://journals.openedition.org/questes/581 ; DOI : https:// doi.org/10.4000/questes.581 


\section{Les épées du roi Arthur}

\section{Patrick MORAN}

De toutes les épées fictives inventées au Moyen Âge, Escalibor est très probablement la plus célèbre, et son association avec le personnage d'Arthur est renforcée dans l'imaginaire collectif par des motifs saisissants et bien connus comme l'épreuve du perron et la main surgie du lac : à un roi extraordinaire correspond une arme également hors du commun, et pour le public du $\mathrm{XXI}^{\mathrm{e}}$ siècle Escalibor se définit avant tout comme l'épée d'Arthur. La situation n'est pourtant pas aussi simple dans les textes médiévaux, notamment pendant la période qui va de Chrétien de Troyes au Merlin post-Vulgate. Si dans le Roman de Brut Calibore est clairement associée à Arthur, dans les textes proprement romanesques qui le suivent il ressort bien plus souvent qu'Arthur n'est pas le seul possesseur d'Escalibor, et inversement qu'Escalibor n'est pas la seule épée qu'Arthur possède. Les épées qui gravitent autour du personnage d'Arthur suivent des cheminements complexes, apparaissant et disparaissant, passant de main en main; cette mobilité intratextuelle se double au demeurant d'une mobilité intertextuelle, le motif de l'épée du roi se modifiant progressivement au fil des romans pour finalement se stabiliser autour d'une seule épée, Escalibor, propriété exclusive et permanente d'Arthur. C'est surtout le Merlin post-Vulgate qui contribuera à cristalliser cette association sous sa forme définitive, marquant en cela sa différence avec le Cycle Vulgate, qui met Escalibor entre de nombreuses mains à tour de rôle et multiplie les épées royales. Dans ce cycle le transfert de l'épée acquiert une valeur symbolique, qu'elle aboutisse entre les mains de Gauvain ou même brièvement entre celles de Lancelot; tandis que le Merlin Huth, tentant de constituer un texte davantage centré sur la figure 
arthurienne (une « Arthuriade», pour reprendre les termes de Fanni Bogdanow ${ }^{1}$ ), verrouille plus fortement le lien entre la figure royale et son arme.

\section{Évolution du motif jusqu'aux textes cycliques.}

Arthur est associé à Escalibor (Calibore) dès le Roman de Brut, qui suit en cela l'Historia regum Britanniae; mais cette association est diluée par le fait que l'épée fait partie d'une triade d'armes, composée également d'un bouclier, Priven, et d'une lance, Roit, eux aussi repris à Geoffroy. Les trois armes sont introduites simultanément, sans que la formulation établisse une quelconque préséance de l'une sur les deux autres. ${ }^{2}$ Dans la suite du récit néanmoins, seule l'épée réapparait ${ }^{3}$ : elle acquiert donc une légère prééminence sur les deux armes, ne serait-ce qu'en raison du nombre plus élevé de ses occurrences.

La liaison initiale entre Arthur et Escalibor ne survit cependant pas au Brut; le passage de la pseudo-chronique guerrière au roman courtois instaure une nouvelle donne qui va contribuer à compliquer la situation. L'épée d'Arthur n'apparaît en effet à aucun moment dans les romans de Chrétien de Troyes, ce qui de prime abord n'a rien de surprenant, puisque les textes de Chrétien se concentrent davantage sur les chevaliers de la cour que sur la figure royale, et que celle-ci n'est jamais présentée sur le mode guerrier qui est l'apanage de la chronique. Pourtant, qu'aucune épée ne soit associée à Arthur ne signifie pas l'absence d'Escalibor; elle est mentionnée une seule fois, dans Le Conte $d u$ graal, entre les mains de Gauvain. Il s'agit de l'épée avec laquelle il défend la sœur du roi de Cavalon contre les citadins en furie :

\footnotetext{
${ }^{1}$ Fanni Bogdanow, The Romance of the Grail, Manchester, Manchester University Press, 1966.

${ }^{2}$ WACE, Arthur dans le roman de Brut, Ivor ARnOld et Margaret PelAn (éd.), Paris, Klincksieck, 1962, v. 733-760.

${ }^{3}$ Ibid., v. 1541, 2997, 4329 et 4348.
} 
Or mes, que qu'an doie avenir,

Cuidera bien contretenir

L'uis et l'antree de la tor,

Qu'il avoit çainte Escalibor,

La meillor espee qui fust,

Qu'ele tranche fer come fust. ${ }^{4}$

L'épisode a beau ne pas être très glorieux, Escalibor n'en demeure pas moins une épée de grande valeur ; mais aucune référence n'est faite à Arthur. L'introduction d'Escalibor dans la littérature vernaculaire, chez Wace et chez Chrétien, se fait donc sur un double mode contradictoire : ou bien Escalibor est l'épée d'Arthur, ou bien elle est l'épée de Gauvain. Dans Arthurian Tradition and Chrétien de Troyes, R. S. Loomis fait remonter cette hésitation sur le propriétaire de l'épée à deux traditions celtiques distinctes ${ }^{5}$. Il constate également que cette incohérence a très tôt gêné les romanciers médiévaux, qui tentent d'y remédier : dès la Première continuation du Conte du graal, le récit introduit une scène de transmission, où Arthur donne son épée Escalibor à Gauvain à l'occasion du combat contre le Riche Soudoier. Le legs temporaire est la solution la plus simple à la contradiction, et le Cycle Vulgate reprend abondamment ce motif par la suite.

\section{Mise en ordre de la matière et pérégrination des épées dans le Cycle} Vulgate.

Le Cycle Vulgate hérite de tous ces éléments, auxquels il faut ajouter le motif de l'épée dans le perron, qui vient du Merlin de Robert de Boron, et sur lequel nous reviendrons. La première base cyclique, autour du Lancelot en prose,

\footnotetext{
${ }^{4}$ Chrétien de Troyes, Le Conte du graal, A. Hilka repris par J. Dufournet (éd.), Paris, GF, 1997, v. 5899-5904.

${ }^{5}$ Roger Sherman Loomis, Arthurian Tradition and Chrétien de Troyes, New York, Columbia University Press, 1949, « Escalibor », p. 421-425.
} 
de La Queste del saint Graal et de La Mort le roi Artu, ne pose pas la question de l'origine d'Escalibor; elle tente avant tout de résoudre les contradictions héritées des deux traditions antérieures. Les solutions qu'elle propose n'apparaissent d'ailleurs que progressivement. Dans le Lancelot, Escalibor n'est encore que le nom de l'épée de Gauvain, comme chez Chrétien. Ce n'est que dans La Mort le roi Artu, lorsque Lancelot et Gauvain s'affrontent devant la cité de Gaunes, que le texte précise en passant qu'il s'agit de l'épée d'Arthur, dont Gauvain a l'usufruit :

[...] et messire Gauvains n'est mie plus lenz, einz cort a son escu qui lui estoit volez del col et met la main a Escalibor, la bone espee le roi Artu, et queurt sus a Lancelot et li done si grant cop seur son hiaume qu'il l'empire et maumet $[\ldots]^{6}$

Le texte reprend la solution déjà esquissée par la Première continuation, même s'il n'explique à aucun moment les circonstances du prêt. En revanche, à la fin de la Mort Artu, alors que Gauvain est déjà mort et qu'Arthur sent approcher sa propre fin, Escalibor est pleinement redevenue l'épée du roi (sans que le texte mentionne à quel moment il a récupéré l'arme) : «Ha ! Escalibor, bone espee et riche, la meilleur de cest siecle, fors cele as Estranges Renges, or perdras tu ton mestre [...]. » ${ }^{7}$

La Suite Vulgate du Merlin, qui se fixe pour but rétrospectif de raconter les événements qui se déroulent entre le couronnement d'Arthur et le début du Lancelot, n'a plus qu'à gommer les dernières aspérités et expliciter pleinement la situation, grâce à une scène où Arthur adoube le jeune Gauvain et lui ceint son épée Escalibor :

\footnotetext{
${ }^{6}$ La Mort le roi Artu, J. FrAPPIER (éd.), Genève, Droz, $3^{\mathrm{e}}$ éd., 1964, § 151.

${ }^{7}$ Ibid., $\S 192$.
} 
Quant vint aprés la messe si prist li rois Artus sa bone espee qu'il osta del perron par le conseil de Merlin, si la chaint a Gavain son neveu au costé $[\ldots]^{8}$

On le voit, le rédacteur de la Suite Vulgate a également choisi d'assimiler Escalibor à l'épée retirée du perron par le jeune Arthur dans le Merlin de Robert de Boron. Le texte de Robert ne donnait aucun nom à l'épée, mais les premières pages de la Suite se chargent de préciser qu'elle en a bien un :

Quant li rois Artus fu desestourdis si traïst l'espee du fuerre qui jeta ausi grant clarté com se .II. cierge i eüssent esté espris. Et ce fu cele espee qu'il ot prise ou perron. Et les letres qui estoient en l'espee escrites disoient qu'ele avoit non Eschalibor. Et c'est un non qui dist en ebrieu «trenche fer et acier et fust $\gg{ }^{9}$

Le rédacteur reprend presque à la lettre la formulation de Chrétien au sujet des propriétés d'Escalibor, en en faisant de surcroît le sens étymologique du nom. Par ce clin d'œil au Conte du graal, il indique qu'il est conscient de la double tradition dont il est redevable; en reprenant les mots de Chrétien à propos de l'épée de Gauvain pour les appliquer à l'épée d'Arthur, il reconnaît la contradiction entre les sources antérieures, contradiction qu'il résout de manière satisfaisante dans la scène de l'adoubement.

Cette solution crée cependant une nouvelle difficulté qui, si elle est marginale, n'en est pas moins réelle : il faut justifier le fait qu'Arthur, en pleine guerre contre ses barons rebelles et contre les Saxons, choisit de se défaire d'une épée aussi remarquable qu'Escalibor, même si c'est pour en ceindre un neveu méritant. La Suite introduit donc une seconde épée fabuleuse, Marmiadoyse, l'arme du roi Rion, chef des envahisseurs qui assiègent Léodagan de Carmélide. Marmiadoyse est si prodigieuse qu'Arthur ne peut s'empêcher de la convoiter lorsqu'il la voit entre les mains de son ennemi :

\footnotetext{
${ }^{8}$ Le Livre du Graal I : Les Premiers faits du roi Arthur, I. FREIRE-NuNES (éd.), Paris, Gallimard, 2001, § 354.

${ }^{9}$ Le Livre du Graal I : Merlin, I. FreIRE-NunEs (éd.), Paris, Gallimard, 2001, § 221.
} 
Quant li rois Rions vit que sa mache fu copee, si traïst l'espee qui tant fu de grant bonté et, si tost com il l'ot jetee hors del fuerre, si rendi si grant clarté qu'il sambla que tous li païs en fust enluminés. Et ele avoit a non Marmiadoyse. Et quant li rois Artus voit l'espee qui si reflamboie, si le proise molt et se traïst un poi en sus pour le regarder, si le couvoite molt durement et dist que bon seroit nés qui le porroit conquerre. ${ }^{10}$

Le texte suggère que Marmiadoyse vaut bien Escalibor, et que l'acquisition de l'une compenserait la perte de l'autre. Arthur parvient à vaincre Rion et lui vole son épée, ce qui permet au rédacteur de lui faire ensuite donner Escalibor à Gauvain sans invraisemblance.

Le Cycle Vulgate reprend donc les deux traditions contradictoires rattachant Escalibor tantôt à Arthur, tantôt à Gauvain, et les concilie par une solution narrative; en même temps, par le truchement du baptême rétroactif de l'épée dans le perron, il offre au lecteur la destinée complète d'Escalibor, de son apparition jusqu'à sa disparition dans le lac. Ce parcours total, fidèle au souci d'exhaustivité de l'écriture cyclique, est également un parcours circulaire: Escalibor, appartenant d'abord à Arthur, puis à Gauvain, avant de revenir à Arthur, est aussi une arme mystérieuse apparue de nulle part et rendue finalement au néant, ou du moins à l'Autre Monde.

Le Cycle Vulgate ayant résolu ces contradictions, il lui reste à conférer un sens au don de l'épée. On serait tenté d'y voir une simple marque d'hérédité et de succession: Gauvain, en tant que neveu préféré d'Arthur, est à bien des égards son héritier naturel; le fait de lui donner Escalibor l'associe en quelque sorte au trône. Mais l'explication par l'hérédité ne justifie pas que l'épée passe brièvement entre les mains de Lancelot, pendant l'épisode de la fausse Guenièvre. Au moment du duel judiciaire opposant Lancelot à trois barons de Carmélide sur la question de l'innocence de la reine, Gauvain, qui est devenu régent, prête Escalibor au héros : «Et quant il fu armés, se li chainst mé sire

${ }^{10}$ Le Livre du Graal I : Les Premiers faits du roi Arthur, op. cit., $\S 305$. 
Gavains Eschalibor, s'espee, et li proie que pour l'amour de lui le port, et il dist qu'il le fera moult volentiers. $\gg{ }^{11}$ Gauvain donne Escalibor à Lancelot pour les mêmes raisons qu'Arthur l'avait ceinte à Gauvain : puisque Gauvain, étant régent, ne peut combattre pour défendre l'honneur de la reine, il fait de Lancelot le champion du royaume en lui prêtant l'épée royale.

La fonction d'Escalibor est d'incarner la puissance royale combattante : ainsi, elle demeure entre les mains d'Arthur pendant la plus grande partie de la Suite Vulgate, puisque le roi mène lui-même ses troupes sur le champ de bataille. Lorsqu'au contraire le régime héroïque cède le pas au régime breton, pour reprendre la terminologie d'Annie Combes ${ }^{12}$, c'est-à-dire, à l'échelle du cycle, lorsque la Suite Vulgate laisse la place au Lancelot, Escalibor devient en propre l'épée de Gauvain. En effet le roi cesse d'avoir un rôle guerrier actif, et le legs de l'arme à son neveu confère à celui-ci un statut de champion du royaume, sorte de bras armé du pouvoir arthurien. L'épée passe ensuite à Lancelot pendant le petit moment où Gauvain est régent. Enfin, lorsque Gauvain meurt et qu'Arthur doit reprendre le commandement actif des armées pour la bataille de Salesbières, l'épée lui revient tout naturellement. Le don d'Escalibor symbolise moins la reconnaissance d'une hérédité que celle d'une légitimité. Le sens de l'épreuve du perron confirme cette symbolique : en retirant l'épée, Arthur ne prouve pas qu'il est le descendant d'Uterpandragon, mais qu'il est l'élu de Dieu ; son succès n'est qu'indirectement la marque de sa véritable hérédité. De même, à sa mort Arthur craint de voir Escalibor tomber entre les mains des «malvés oir »; seul Lancelot, malheureusement absent, aurait été le digne récipiendaire de l'arme miraculeuse - alors même qu'il n'entretient aucun lien de parenté avec Arthur.

\footnotetext{
${ }^{11}$ Le Livre du Graal II : Lancelot, M. Demaules (éd.), Paris, Gallimard, 2003, « Galehaut », $\S 112$.

${ }_{12}$ Annie COMBES, Les Voies de l'aventure. Réécriture et composition romanesque dans le Lancelot en prose, Paris, Champion, 2001, « Des types d'écriture diversifiés », p. 97-105.
} 


\section{La solution alternative du Merlin Huth et ses conséquences.}

Le Cycle Vulgate offre un discours cohérent et élaboré sur l'épée d'Arthur ; Escalibor, symbole de la légitimité du pouvoir royal, circule de main en main selon ce qu'exigent les circonstances, pour revenir toujours in fine à Arthur, et finalement disparaître avec lui. La seule incohérence résiduelle réside précisément dans cette disparition mystérieuse : rien n'explique comment Arthur peut savoir qu'une main surgira du lac pour attraper l'épée. Le Merlin Huth essaie de lisser cette dernière aspérité, et ce faisant bâtit sa propre version d'Escalibor.

Le Merlin Huth s'inscrit dans le projet de ce que Fanni Bogdanow appelle une «Arthuriade», en concurrence directe avec le Cycle Vulgate qui accordait la part belle aux chevaliers, Lancelot au premier chef. La première conséquence de cette réorientation, c'est qu'Escalibor devient la propriété exclusive d'Arthur : nul autre que lui ne peut la posséder, toute circulation de l'arme devient illégitime. Le motif se rigidifie et se lie fermement au personnage du roi. Cependant Escalibor n'est plus l'épée dans le perron : le rédacteur du Merlin Huth, pour rationaliser la disparition mystérieuse de l'épée à la mort du roi, construit une scène symétrique où Merlin mène le jeune Arthur auprès d'un lac, d'où surgit une main qui brandit l'arme. L'épée du perron perd donc toute valeur autre que symbolique : sans autre but que de signifier l'élection divine d'Arthur, elle ne dispose d'aucune vertu supplémentaire. C'est d'ailleurs parce qu'Arthur la brise en combattant Pellinor que Merlin le mène au lac afin de remplacer son arme :

«A poi, fait li rois, que je n'ai esté hounis par m'espee qui me failli. - Ne le vous disoie je bien, fait Merlins, qu'elle ne vous averoit ja duree? Et sacés que je ne sai en che païs c'une boine espee, et cele est en un lach ou fees habitent. Se celle poés avoir, elle vous durroit tres qu'a la fin. ${ }^{13}$

\footnotetext{
${ }^{13}$ La Suite du roman de Merlin, Gilles RoussineAu (éd.), Genève, Droz, 1996, § 61.
} 
Les propos de Merlin sont explicites: la possession d'Escalibor dispensera Arthur de chercher d'autres épées, puisque celle-ci est si excellente qu'elle l'accompagnera jusqu'à sa mort. Quelques résidus de prolifération subsistent cependant dans le Merlin Huth. Arthur possède une première épée avant d'acquérir Escalibor. De plus, si Escalibor est une épée hors du commun, elle est moins remarquable que son fourreau, dont les pouvoirs sont encore plus considérables, explique Merlin :

«Saichiés vraiement que li fuerres vaut miex que teuls .C. espees ne font, car il est d'un cuir qui a tel viertu que ja hom qui sour lui le porte ne perdera sanc ne ne rechevra ja plaie mortel, pour qu'il soit armés a raison. $\gg{ }^{14}$

Ainsi, bien qu'Arthur ne possède plus qu'une seule épée vraiment remarquable, elle est double. Autour de ce fourreau magique viennent d'ailleurs s'agencer plusieurs complications narratives; Morgain tente de voler les deux objets à plusieurs reprises, et l'épée se retrouve finalement séparée du fourreau après le duel d'Arthur contre l'amant de Morgain, Accalon. La « transmission » des deux objets se réduit au motif du vol et de l'appropriation illégitime. Le fourreau problématique finit de toute façon par disparaître, jeté dans un lac par Morgain : plus personne ne le reverra ni ne bénéficiera de ses pouvoirs, si ce n'est Gauvain - retour inattendu et fugace du motif du prêt de l'épée - qui en disposera une seule fois, lorsqu'il affrontera l'enchanteur Mabom. ${ }^{15}$

Hormis ce duel que le texte annonce sans jamais le relater, l'équation se réduit, avec la perte du fourreau, à un seul homme et à une seule épée, indissolublement liés. Le motif de l'épée d'Arthur s'est durci, tout en devenant progressivement plus cohérent et plus exclusif; Escalibor passe de l'état transmissible à l'état intransmissible. Elle n'est plus le symbole d'hérédité ou de

\footnotetext{
${ }^{14}$ Ibid., $\S 65$.

${ }^{15}$ Ibid., § 413.
} 
légitimité qu'elle était dans le Cycle Vulgate : elle sert plutôt à souligner la spécificité irréductible d'Arthur, roi qui n'a son pareil à aucune époque de l'histoire de l'humanité. Le fait qu'elle soit dissociée de l'épée dans le perron efface les dernières traces d'hérédité biologique qui pouvaient encore s'y rattacher. Les deux scènes du lac, celle où le jeune roi obtient Escalibor et celle où il la rend aux flots, marquent une parenthèse fabuleuse dans l'histoire de Bretagne, centrée autour d'un seul homme qui semble être, à l'instar de son épée, sui generis. 